\title{
EL MOVIMIENTO NATURAL DE LA POBLACION DE ESPAÑA
}

$325.331(46)$

por

Ignacio Ballester Ros

SUMARIO: I. INTRODUCCION.-II. EL MOVIMIENTO NATURAL DE LA POBLACION DE ESPAÑA EN LAS PROVINCIAS Y EN LAS CAPITALES DE PROVINCIA: 1. LA NUPCIALIDAD. 2. LA NATALIDAD. 3. LA MORTALIDAD.-III. EL CRECIMIENTO VEGETATIVO EN LAS PROVINCIAS Y EN LAS CAPITALES DE PROVINCIA.-IV. APENDICE ESTADISTICO.

\section{INTRODUCCION}

La "Estadística del Movimiento Natural de la Población”, juntamente con el "Censo General de la Población", constituyen las. dos investigaciones estadísticas oficiales más antiguas de España realizadas por el Instituto Nacional de Estadística o los organismos que le predecieron en estas tareas.

Mientras que el primer "Censo Oficial de la Población" se realiza, en 1857, por la Comisión de Estadística General del Reino, el "Movimiento Natural de la Población" es la primera estadística llevada a cabo, en 1858, por la Junta General de Estadística del Reino. 
En una primera etapa, a partir de 1858 , los datos primarios relativo a los fenómenos básicos del movimiento natural de la población-matrimonios, nacimientos, defunciones-se toman de los Registros parroquiales. Al promulgarse la Ley provisional del Registro Civil de 17 de junio de 1870, se organizan las correspondientes Oficinas en los Juzgados y los datos se toman ya de las actas de inscripción en los libros del Registro Civil mediante boletines especiales.

Durante más de un siglo han sido los Secretarios de los Ayuntamientos, como encargados al propio tiempo de las secretarías de los Juzgados municipales-también denominados de paz en algunos períodos-, quienes suministran a las Delegaciones provinciales de Estadística la información referente a actos realizados e inscritos en los libros correspondientes, referentes a los Municipios radicados en el amplia área rural de España de hasta 7.000 habitantes últimamente.

La primera monografía sobre el "Movimiento Natural de la Población" se publica en 1863 y comprende los datos de 1858 a 1861 . Los datos del decenio 1861-1870 se publican en un solo volumen y los de 1886-1890 en otro. A partir de 1900 se publican en volúmenes anuales, con algunas excepciones. No se publicaron en monografías propias los datos correspondientes a los años 1871 a 1885 y de 1893 a 1899.

Durante mucho tiempo la explotación estadística de los datos se realizó por procedimientos manuales. En 1968 se realizan los primeros ensayos de mecanización, aplicada a los boletines de defunción. La mecanización de los datos de matrimonios y de nacimientos se lleva a cabo con posterioridad, una vez resueltos algunos problemas conceptuales.

A partir de 1975 se elabora esta estadística con una notable ampliación de las clasificaciones relativas a las características de los fenómenos, de forma similar a la de los países europeos. Se ha procedido a una revisión total de la misma, que afecta a los conceptos básicos, a los boletines empleados, a las tablas de resultados y a los procedimientos de explotación. Además de los matrimonios actualmente se contemplan los nacimientos y, como su complemento, los partos y las muertes fetales tardías, así como las defunciones, con una especial atención al análisis de las causas de muerte.

Dado el hecho de que las maternidades y otros centros sanitarios de capitales de Provincia y grandes Municipios atienden a madres y otros enfermos residentes en otros Municipios de la Provincia, en 
especial en los colindantes y periféricos de los primeros, se ha establecido como variable a tener en cuenta la residencia de la madre en los nacimientos y, en su caso, la del difunto, al objeto de corregir la asignación tradicional del hecho producido al Municipio en que ha tenido lugar, atribuyéndolo al Municipio de residencia del causante.

Al objeto de profundizar en el estudio de la fecundidad, se investiga la edad de la madre en el momento del nacimiento del hijo, el orden de nacimiento en el conjunto de hijos habidos por ella y su intervalo genésico.

El plan de publicaciones del Instituto Nacional de Estadística, referente a esta investigación, es también nuevo. La monografía anual comprende, desde 1975, tres tomos. El primero está dedicado a las cifras nacionales y a algunos resúmenes provinciales, relativos a los hechos registrados. El segundo comprende nueve volúmenes, que contienen una amplia información sobre las características de los hechos estudiados por Provincias. capitales de Provincia y cada uno de los Municipios mayores de 10.000 habitantes, con un resumen de los fenómenos básicos, con referencia a cada uno de los Municipios españoles. El tercero contiene el análisis de las causas de muerte, según las clasificaciones internacionales de la Organización Mundial de la Salud. También ha publicado dicho Instituto un folleto sobre "Dinámica de la población española", en el que se examina la evolución y características de los matrimonios, nacimientos y defunciones en los últimos años.

La evolución registrada por la "Estadística del Movimiento Natural de la Población", según se ha descrito en los párrafos anteriores, con arreglo a las recomendaciones de la Comisión de Estadística de las Naciones Unidas sobre estadísticas vitales, su carácter más que centenario y la importancia que tiene desde el punto de vista local hacen aconsejable dedicarle el presente comentario.

El estudio del crecimiento vegetatitvo-diferencia entre las cifras de nacimientos y de defunciones-, como colofón de los hechos antes referidos, resulta esencial y que, conjuntamente con los movimientos migratorios, influyen decisivamente en la evolución de la población en las distintas áreas territoriales y, consiguientemente, en su desarrollo demográfico, económico y social.

Limitaremos, sin embargo, este trabajo simplemente al análisis de las cifras globales de matrimonios, nacimientos y defunciones, y al crecimiento vegetativo y sus respectivas lasas en las Provincias y (ap)itales de Provincia en 1978. 


\section{EL MOVIMIENTO NATURAL DE LA POBLACION DE ESPAÑA EN LAS PROVINCIAS Y EN LAS CAPITALES DE PROVINCIA}

En las tablas I y II del Apéndice Estadístico se insertan las cifras absolutas, expresivas de los matrimonios, nacimientos y defunciones habidas en el año 1978, y en las tablas III y IV se presentan los respectivos coeficientes por mil habitantes.

Seguidamente analizaremos la distribución de estos hechos, según su grado de intensidad, en las Provincias y en las capitales de Provincia, con un somero comentario a manera de conclusiones.

\section{LA NUPCIALIDAD}

Puede afirmarse que la nupcialidad sólo presenta pequeñas fluctuaciones de carácter coyuntural a lo largo del presente siglo. Aumenta, en cifras absolutas, el número de matrimonios, paralelamente al incremento de la población; pero se mantiene la tasa por mil habitantes dentro de unos límites próximos entre sí, aunque con una constante tendencia a la baja. En 1900 dicha tasa fue de 8,67 matrimonios por mil habitantes, y en 1978 ha sido de 6,94.

\section{a) Por Provincias}

Al analizar su distribución provincial se observa, en primer término, que el rango o diferencia-2,78-entre el valor mínimo $-5,51-$, correspondiente a Guadalajara, y el máximo-8,29-, de Cáceres, es reducido. La dispersión de valores alrededor de la tasa nacional es igualmente reducida, 1,35 respecto al valor máximo y 1,43 en relación al valor mínimo.

A 31 se elevan las Provincias con tasas superiores a la nacional, las cuales se aprupan geográficamente en esta forma:

- Galicia y Cantábrico: La Coruña, Lugo, Pontevedra y Santander.

- Valle del Duero: León, Zamora, Salamanca, Palencia y Segovia.

- Submeseta Sur: Cáceres, Badajoz, Toledo y Albacete.

- Valle del Ebro: Navarra, Logroño y Zaragoża.

- Litoral catalán-levantino: Gerona, Tarragona, Castellón, Valencia, Alicante y Murcia.

- Andalucía: Todas sus Provincias.

- I.as Palmas.

Se registra un predominio de Provincias predominantemente agrarias en cultivo extensivo, las más, y en cultivo intensivo, algu- 
nas también con una importante actividad industrial. Los valores máximos corresponden a Cáceres, Gerona y León.

Salvo en Andalucía, en las demás zonas consideradas coexisten Provincias de tasas más altas con otras de tasas más bajas que la nacional. Y surgen las Vascongadas, con tasas bajas.

Resulta evidente que los movimientos migratorios interiores influyen en la evolución de la nupcialidad. El envejecimiento relativo de la población de Provincias con mayor proporción de personas adultas, debido a que la población más joven ha emigrado, repercute en la existencia de bajas tasas de nupcialidad, como ocurre en Guadalajara, Huesca y Teruel. También se observan dichas tasas, sin embargo, en Provincias de gran desarrollo industrial, como Barcelona y Guipúzcoa, o predominantemente turísticas, como Baleares, aunque este fenómeno debe obedecer a otras causas, que se manifiestan en el entorno social, que influye al dar nacimiento a una tendencia hacia las uniones extramatrimoniales.

\section{b) Por capitales de Provincia}

La nupcialidad en el conjunto de capitales de Provincia es ligeramente superior a la de las Provincias. La diferencia entre los valores mínimos, correspondientes a Barcelona y Gerona, y los - máximos, de Zamora y Avila, es más elevada, y la dispersión de valores es más pronunciada y tiende hacia los valores máximos.

Las capitales con coeficientes superiores al nacional son 29, y configuran estas zonas geográficas:

- Galicia y Cantábrico: Lugo, Orense y Oviedo.

- Valle del Duero: León, Zamora, Salamanca, Burgos, Soria, Segovia y Avila.

- Submeseta Sur: Cáceres, Badajoz, Toledo, Cuenca, Guadalajara y Albacete.

- Valle del Ebro: Huesca, Zaragoza, Teruel y Lérida.

- Litorial oriental mediterráneo: Palma de Mallorca y Murcia.

- Andalucía: Todas las capitales, excepto Huelva.

La capital de España, Madrid, así como Valladolid y Valencia, se hallan muy próximas al coeficiente nacional.

Existe una gran similitud entre estas zonas configuradas por las capitales y las de las Provincias, aunque difieren parcialmente. La diferencia es más acusada en el litoral mediterráneo oriental.

Las tasas de nupcialidad superiores a la nacional predominan en las ciudades pequeñas y de tamaño medio de la gran zona agraria 
española, si bien se registran también en ciudades ubicadas en las zonas industriales.

\section{LA NATALIDAD}

Los países europeos estuvieron sometidos hasta el siglo XVIII a altas tasas de natalidad y también a una mortalidad infantil muy elevada.

A lo largo del siglo XVIII y la primera mitad del siglo XIX mejoran las condiciones médicas e higiénicas, crece la producción agricola, resultan positivos los efectos de la revolución industrial, la alimentación, el alojamiento y vestido evolucionan y permiten un más alto nivel de vida, que se pone abiertamente de manifiesto hacia 1850. Disminuye la mortalidad infantil, se produce un excedente de hijos, se registra un incremento de la población, se eleva la vida media del hombre y se inicia un descenso de la natalidad

En el siglo $\mathrm{XX}$ se acelera el decrecimiento de la natalidad. La urbanización progresiva, los movimientos migratorios desde el campo hacia las grandes urbes y a las ciudades industriales produce un alejamiento de los valores tradicionales y el debilitamiento de los sentimientos religiosos. La aceptación de los métodos anticonceptivos y una tendencia hacia una vida más cómoda en las familias conduce a un notable decrecimiento de la natalidad.

Las mismas causas expuestas influyen en el decrecimiento de la natalidad española. El número de nacimientos se mantiene en unos volúmenes parecidos desde principios de siglo; pero, como la población aumenta desde 18,5 millones de habitantes en 1900 a 37 millones en 1978, las tasas de natalidad registran una notable disminución. En 1900 era de 33,8, y en 1978 se había reducido a la mitad, a 17,66 .

\section{a) Por Prozincias}

La diferencia entre los valores mínimos, correspondientes a Soria y Teruel, y los máximos, de Cádiz y Sevilla, supera los 11 enteros. La dispersión de los valores tiende más hacia los mínimos.

Tan sólo 17 Provincias presentan tasas de natalidad superiores a la nacional, y se distribuyen geográficamente de esta manera:

- Calicia y Cantábrico: Ponteredra : Santander.

- Valle del Ducro: Valladolid.

- Submesera Sur: Madrid y Albacele.

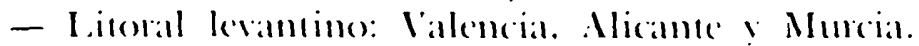


- Andalucia: Todas las Provincias, salvo Jaén.

- Ceuta y Las Palmas.

Resulta indicativo, en primer término, que frente a 31 Provincias con altas tasas de nupcialidad, sólo se registren 17 con altas tasas de natalidad. Ello corrobora la existencia de una natalidad verdaderamente decreciente.

Como Regiones, solamente Andalucía y Levante destacan por su natalidad favorable, y junto a ellas, Provincias aisladas en la Meseta, al Norte, y en Canarias.

La baja natalidad cubre prácticamente la Meseta, Galicia y la Orla Cantábrica, el Valle del Ebro y Cataluña. Cubre tanto grandes Regiones industriales como las zonas agrícolas, tanto de cultivo extensivo como intensivo. Se trata, pues, de un fenómeno muy generalizado.

Si bien Barcelona, Alava y Vizcaya están cerca de la tasa nacional, otras Provincias, de predominio agrario, figuran con las tasas mínimas, junto con Soria y Teruel. Son Lugo y Orense, Huesca, Zamora, Avila y Segovia, Cáceres, Cuenca y Guadalajara, la mayor parte de ellas situadas en la Meseta.

Una baja nupcialidad, por las causas apuntadas en el apartado anterior, unida a las causas intrínsecas de disminución de la natalidad, también expuestas, determinan una tendencia negativa de la natalidad.

\section{b) Por capitales de Provincia}

La tasa de natalidad de las capitales es, en un 70 por 100, más elevada que la de las Provincias, y de manera similar a lo que ocurre con la nupcialidad, aunque más acentuadamente, la diferencia entre los valores mínimos, correspondientes a Lugo, Palma de Mallorca y Bilbao, y lús máximos, propios de Toledo y Ciudad Real, es muy elevada, y también la dispersión de los valores es más acusada y tiende hacia los valores máximos.

Las capitales con coeficiente superior al nacional son 30 , y se distribuyen en la siguiente forma:

- Cantábrico: Santander y San Scbastián.

- Valle del Duero: Zamora, Salamanca, Palencia, Soria, Segovia y Avila.

- Submeseta Sur: Cáceres, Badajor., Tolcelo, Ciudad Real, Cuenca, Guadalajara y Albacete. 
- Valle del Ebro: Pamplona, Logroño, Huesca, Teruel y Lérida.

- Región valenciana: Castellón, Valencia y Alicante.

- Andalucía: Todas las capitales, excepto Córdoba.

Esta distribución de capitales resulta bastante similar a la de la nupcialidad, singularmente en Andalucía, la Meseta y el Valle del Ebro. Difieren sustancialmente en el Cantábrico y, sobre todo, en la Región valenciana.

Una vez más, estas tasas superiores a la nacional predominan, al igual que se daba con la nupcialidad, en las ciudades pequeñas y de tamaño medio de la gran zona agraria española y en algunas, pocas, ciudades industriales.

Junto a las capitales con tasas mínimas aparecen otras, con tasas más elevadas, pero todavía inferiores a la nacional, que cubren las Regiones de Galicia y Asturias, Cataluña y Canarias, y parcialmente los Valles del Duero y Ebro, además de otras aisladas, como Córdoba y Murcia, y, con carácter excepcional, Madrid, que, junto con Barcelona, las dos grandes urbes españolas, ofrecen una débil natalidad.

\section{LA MORTALIDAD}

El descenso de la mortalidad precede históricamente al de la natalidad. En primer término, y tras un largo proceso, disminuye la mortalidad infantil en toda Europa Occidental, y con un cierto desfase en el tiempo, en España, donde alcanzaba, en 1900, a 205 menores de un año por cada mil nacidos, y en 1975 queda reducida al 19 por 1.000 .

También se reduce la mortalidad general. En cierto modo hay unas concausas que influyen, al propio tiempo, en el decrecimiento de la natalidad y de la mortalidad. Entre ellas figuran los avances de la Medicina, la política sanitaria llevada a cabo con tanta eficacia por Europa Occidental, el aumento de la producción agrícola y del nivel de vida, que ha determinado la prolongación de la vida y, consiguientemente, el envejecimiento de la población.

La mortalidad general ha seguido también en España una tendencia descendente. Desde una tasa de 27,7 fallecidos por mil habitantes a principios de siglo, bajó al 23 en 1920, al 15,6 en 1935, al 10,8 en 1950 y al 7,8 en 1978 .

Al propio tiempo, la vida media del español, que era de cuarenta y siete años en 1900, llega a setenta y cuatro en los tiempos actuales. 


\section{a) Por Provincias}

La diferencia entre las tasas mínimas, correspondientes a Guipúzcoa y las dos Provincias canarias, y las máximas, de Lugo y Teruel, supera los 6 enteros, y la dispersión de los valores tiende más hacia los máximos.

En este caso, las tasas mínimas son las más significativas. Sólo 13 Provincias presentan tasas de mortalidad inferiores a la nacional y configuran estas zonas:

- Galicia y Orla Cantábrica: Pontevedra, Santander y las Vascongadas.

- Meseta: Valladolid y Madrid.

- Barcelona.

- Cádiz.

- Ceuta, Melilla y Canarias.

Resulta paradójico que el número de Provincias con baja mortalidad sea aún más reducido que el de Provincias con altas tasas de natalidad.

Como se advierte, la baja mortalidad corresponde esencialmente a las grandes urbes y a las principales zonas industriales.

Próximas a la tasa nacional, aunque más altas, son las tasas de Oviedo, Navarra, Zaragoza y Huesca; Burgos; Baleares, Valencia, Alicante y Murcia; Sevilla, Málaga, Córdoba, Granada, Jaén y Almería, que amplían las zonas consideradas.

Las 24 restantes Provincias, con las tasas más elevadas de mortalidad, se hallan situadas primordialmente en la Meseta, con derivaciones hacia Galicia, el Valle del Ebro y parte de Cataluña y Andalucía.

\section{b) Por capitales de Provincia}

La tasa de mortalidad de las capitales es ligeramente superior a la de las Provincias. La diferencia entre los valores mínimos, correspondientes a las ciudades de Valladolid, Cáceres y Las Palmas, y los máximos, de Avila y Oviedo, supera los 10 enteros. La dispersión de valores, mayor que la de las Provincias, tiende igualmente hacia los valores máximos.

Las capitales con coeficiente inferior al nacional son 15 y se distribuyen geográficamente en estas zonas:

- Galicia y Orla Cantábrica: La Coruña y Bilbao.

- Valle del Duero: Burgos, Zamora y Valladolid. 
- Submeseta Sur: Madrid, Cáceres y Badajoz.

- Valle del Ebro: Vitoria, Zaragoza, Huesca, Lérida y Tarragona.

- Canarias. Las Palmas y Santa Cruz.

Si comparamos esta distribución con la de por Provincias, advertimos ciertas diferencias. Aparecen varias capitales del Valle del Ebro, se ensancha la zona de la Meseta y desaparecen Barcelona y Cádiz.

Por encima de la tasa nacional, aunque próximas a ella, figuran: Pontevedra; Soria, León y Segovia; Albacete, Cuenca y Guadalajara; Logroño, Alicante y Barcelona; Córdoba, Málaga y Sevilla, que igualmente ensanchan las zonas consideradas.

Entre las 22 capitales restantes figuran grandes ciudades, como Valencia; ciudades de tamaño pequeño y medio, sitas en la Meseta, en Andalucía y al Norte, muchas de ellas de economía predominantemente agraria y algunas en el centro de importantes focos industriales.

\section{EL CRECIMIENTO VEGETATIVO EN LAS PROVINCIAS $Y$ EN LAS CAPITALES DE PROVINCIA}

La diferencia entre la cifra de nacimientos y la de defunciones expresa el crecimiento vegetativo de un área territorial en un período determinado.

Del volumen de crecimiento vegetativo que tenga un país, es decir de su potencial demográfico, depende en gran manera su desarrollo económico. En estos últimos decenios hemos asistido al fenómeno de los desplazamientos de población de los países mediterráneos a los de Europa Central y Occidental en cifras muy importantes. Si de una parte denotan la existencia de fuertes contingentes humanos que no encuentran empleo en sus países de origen, debido a su insuficiente desarrollo económico, desde otra perspectiva es un claro exponente de que los países receptores tienen un débil crecimiento vegetativo, por su solo movimiento natural de la población, y necesitan de mano de obra extranjera para atender al mantenimiento y desarrollo de su actividad económica. Uno de los factores básicos del desarrollo económico lo constituye el factor humano, y sin una base humana suficiente, sin una mano de obra de un cierto volumen y de un grado de especialización adecuado, no puede acometerse el desarrollo económico de un país. De ạpui la importancia que liene el crecimiento vegetativo de los paíse's. 
La tasa española de crecimiento vegetativo puede considerarse normal, ya que desde principios de siglo viene oscilando entre el 12 por 1.000 como máximo y el 7 como mínimo, a excepción de los años 1918 y 1919 , con la epidemia gripal, y de 1937 a 1939, con la guerra civil, que determinaron una elevada mortalidad y un crecimiento vegetativo muy reducido.

Analizando los coeficientes por mil habitantes de la tabla III, se observa que el crecimiento vegetativo en 1978, para el conjunto de España, no llega al 10 por 1.000 , que es escaso, y similar a los países de Europa Occidental.

\section{a) Por Provincias}

La distribución por Provincias es muy significativa, en orden al futuro de la población en dichas áreas.

Tan sólo 20 Provincias presentan una tasa de crecimiento vegetativo superior a la nacional. Son mayoría las que tienen tasas inferiores, algunas con valores por debajo del 1 por 1.000 , y dos de ellas, Lugo y Teruel, con un crecimiento vegetativo negativo, lo que equivale a una disminución de la población.

Los valores oscilan en dicha tabla entre un máximo correspondiente a Cádiz y Sevilla; y los valores negativos señalados. Ello indica que la dispersión de valores es muy amplia y que, lógicamente, este fenómeno no se presenta con la homogeneidad con que, dentro de ciertos límites, ofrece la nupcialidad.

Las Provincias con crecimiento vegetativo superior a la tasa nacional se distribuyen geográficamente en estas zonas:

- Galicia y Orla Cantábrica: Pontevedra, Santander, Vizcaya y Alava.

- Meseta: Valladolid y Madrid.

- Litoral mediterráneo oriental: Barcelona, Valencia, Alicantte y Murcia.

- Andalucía: Todas las Provincias, a excepción de Jaén.

- Ceuta y Canarias.

A ellas cabría añadir, incluida en la primera zona, a Guipúzcoa, con una tasa muy próxima a la nacional.

Esta distribución se parece mucho a la de la natalidad por Provincias. Se advierte un predominio de Provincias con grandes urbes, de gran desarrollo industrial y con áreas agrícolas de cultivo intensivo. Casi todas estas Provincias se hallan en la periferia. Sorprende 
el crecimiento vegetativo andaluz, que denota la existencia de una reserva demográfica notable.

Las 31 Provincias restantes, incluidas Ceuta y Melilla, presentan una amplia gama de tasas, desde las negativas ya apuntadas, y las mínimas al 1 por 1.000 , a las que se acercan a la tasa nacional.

Entre ellas son de señalar, con tasas de hasta el 3 por 1.000 , once Provincias, agrupadas en esta forma:

- Galicia: Lugo y Orense.

- Centro: Zamora, Palencia, Avila, Segovia, Soria, Guadalajara, Cuenca y Teruel.

- Huesca.

De ello se deduce que las tasas inferiores a la nacional se extienden preferentemente por la Meseta; y los valores más bajos de entre ellos se sitúan en el Valle del Duero y el Sistema Ibérico, en la Galicia Mesetaria y en el Ebro Central.

\section{b) Por capitales de Provincia}

El crecimiento vegetativo del conjunto de capitales es muy superior al de las Provincias, al que más que duplica, según se desprende de la observación de la tabla IV.

La oscilación de valores entre las capitales es también acentuada, desde unos mínimos, correspondientes a Lugo y Palma de Mallorca, a las más elevadas de Toledo o Ciudad Real.

Con valores superiores a la tasa nacional se hallan 30 capitales, que configuran las siguientes zonas:

- Orla Cantábrica: Santander.

- Valle del Duero: Zamora, Salamanca, Palencia, Avila, Segovia y Soria.

- Submeseta Sur: Cáceres, Badajoz, Toledo, Ciudad Real, Cuenca, Guadalajara y Albacete.

- Valle del Ebro: Pamplona, Logroño, Huesca, Teruel y Lérida.

- Litoral mediterráneo oriental: Tarragona, Castellón, Alicante y Valencia.

- Andalucía: Todas las capitales, salvo Córdoba.

Se observa, en general, un predominio de las ciudades castellanas y aragonesas, catalanas y valencianas, así como andaluzas, de tamaño medio, y también grandes ciudades, como Valencia y Sevilla. 
ESTADISTICA

\section{APENDICE ESTADISTICO}

1. MOVIMIENTO NATURAL DE LA POBLACION Provincias. 1978

\begin{tabular}{|c|c|c|c|c|}
\hline PROV'INCIAS & Marrimonios & Nacimientos & Defunciones & $\begin{array}{l}\text { Crecimiento } \\
\text { regetativo }\end{array}$ \\
\hline 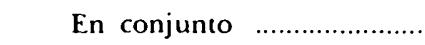 & 257.395 & 632.975 & 290.042 & 342.933 \\
\hline Alava & 1.700 & 4.182 & 1.658 & 2.524 \\
\hline Albacete ................................... & 2.506 & 5.958 & 3.084 & 2.874 \\
\hline 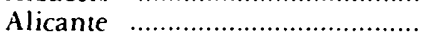 & 8.043 & 20.173 & 9.091 & 11.082 \\
\hline Almería & 2.902 & 7.685 & 3.281 & 4.404 \\
\hline Avila ....................................... & 1.169 & 2.118 & 1.751 & 367 \\
\hline Badajoz …… & 4.467 & 10.217 & 6.248 & 3.969 \\
\hline Baleares & 3.852 & 9.896 & 5.377 & 4.519 \\
\hline Barcelona .................................. & 27.098 & 74.795 & 29.392 & 45.403 \\
\hline 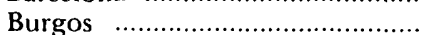 & 2.305 & 5.043 & 2.784 & 2.259 \\
\hline 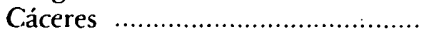 & 3.398 & 5.700 & 3.842 & 1.858 \\
\hline 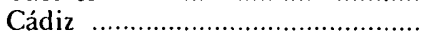 & 7.828 & 21.282 & 6.840 & 14.442 \\
\hline 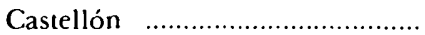 & 3.221 & 6.693 & 4.208 & 2.485 \\
\hline 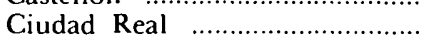 & 3.192 & 7.269 & 4.460 & 2.809 \\
\hline 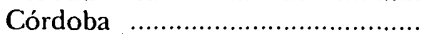 & 5.124 & 12.546 & 5.819 & 6.727 \\
\hline 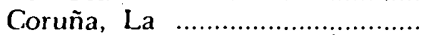 & 7.744 & 17.920 & 9.561 & 8.359 \\
\hline Cuenca ................................... & 1.458 & 2.526 & 2,201 & 325 \\
\hline 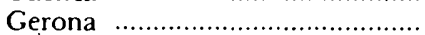 & 3.774 & 7.472 & 4.389 & 3.083 \\
\hline Granada & 5.497 & 13.698 & 5.923 & 7.775 \\
\hline 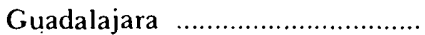 & 746 & 1.771 & 1.281 & 490 \\
\hline 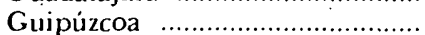 & 4.029 & 10.570 & 4.063 & 6.507 \\
\hline Huelva & 3.087 & 7.643 & 3.899 & 3.744 \\
\hline Huesca & 1.239 & 2.413 & 1.871 & 542 \\
\hline 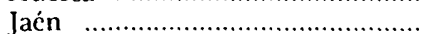 & 4.847 & 10.243 & 5.192 & $5.05 \mathrm{l}^{\circ}$ \\
\hline León …… & 4.132 & 7.241 & 4.732 & 2.509 \\
\hline Lérida & 2.416 & 5.248 & 3.429 & 1.819 \\
\hline 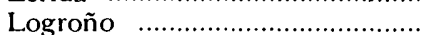 & 1.826 & 3.952 & 2.187 & 1.765 \\
\hline Lugo & 2.782 & 4.725 & 4.767 & -42 \\
\hline 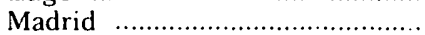 & 30.275 & 85.141 & 27.699 & 57.442 \\
\hline 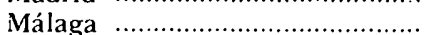 & 6.840 & 18.357 & 7.657 & 10.700 \\
\hline 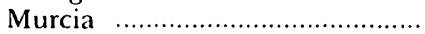 & 6.932 & 19.222 & 7.558 & 11.664 \\
\hline Navarra & 3.533 & 7.793 & 4.221 & 3.572 \\
\hline Orense & 2.584 & 4.675 & 4.293 & 382 \\
\hline Oviedo …….............................. & 7.757 & 16.383 & 9.399 & 6.984 \\
\hline 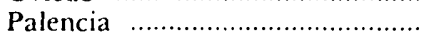 & 1.437 & 2.535 & 1.824 & 711 \\
\hline 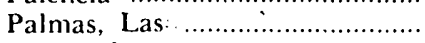 & 5.424 & 13.772 & 4.362 & 9.410 \\
\hline Pontevedra ............................... & 6.214 & 16.012 & 6.717 & 9.295 \\
\hline Salamanca ……....................... & 2.666 & 5.033 & 3.232 & 1.801 \\
\hline Santa Cruz de Tenerife ............ & 4.584 & 11.918 & 4.421 & 7.497 . \\
\hline 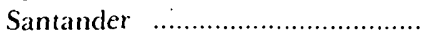 & 3.876 & 8.709 & 3.890 & 4.819 \\
\hline 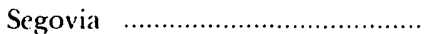 & 1.147 & 1.969 & 1.399 & 570 \\
\hline Sevilla ....................................... & 11.107 & 30.872 & 11.017 & 19.855 \\
\hline 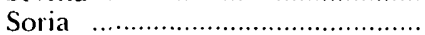 & 612 & 1.065 & 1.000 & 65 \\
\hline Tarragona ….............................. & 3.572 & 8.627 & 4.848 & 3.779 \\
\hline Тетие & 88.1 & 1.616 & .1 .668 & -52 \\
\hline Toledo ...1. & 3.423 & 7.230 & 4.363 & 2.867 \\
\hline 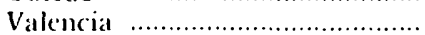 & 14.996 & 37.295 & 17.943 & 19.352 \\
\hline Valladolid ................................... & 3.159 & 8.467 & 3.150 & 5.317 \\
\hline 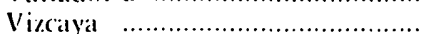 & 7.770 & $20 .+21$ & 7.893 & 12.528 \\
\hline 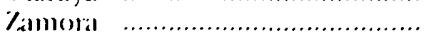 & l.(i2)3 & 2.68 .1 & 2.398 & 350 \\
\hline 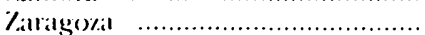 & 5.856 & 12.122 & 6.911 & 5.211 \\
\hline 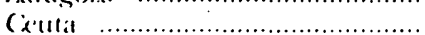 & 117 & 1.237 & 500 & $7: 37$ \\
\hline - & 325 & 8.11 & 119 & 192 \\
\hline
\end{tabular}


REVL-1980, núm. 205. BALLESTER ROS, IGNACIO. EL MOVIMIENTO NATURAL DE LA POBLACI...

REVISTA DE ESTUDIOS DE LA VIDA LOCAL

2. MOVIMIENTO NATURAL DE LA POBLACION

Capitales de Provincia. 1978

\begin{tabular}{|c|c|c|c|c|}
\hline (APITALES DE PROVINCIA & Marrimonios & Nacimiemess & Derfunciones: & $\begin{array}{l}\text { (eccimicento } \\
\text { vegelativos }\end{array}$ \\
\hline 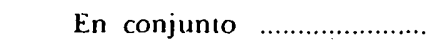 & 98.964 & 394.971 & 120.152 & 274.819 \\
\hline Vitoria & 1.288 & 3.990 & 1.337 & 2.65 .3 \\
\hline 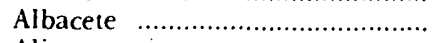 & 809 & 3.340 & 950 & 2.390 \\
\hline Alicante & 1.630 & 10.303 & 2.335 & 7.968 \\
\hline 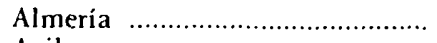 & 1.358 & 6.092 & 1.311 & 4.781 \\
\hline Avila & 522 & 1.834 & 617 & 1.271 \\
\hline 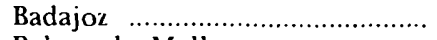 & 852 & 4.395 & 1.210 & 3.185 \\
\hline Palma de Mallorca ........................ & 1.984 & 5.467 & 2.456 & 3.011 \\
\hline 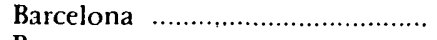 & 9.592 & 42.868 & 16.448 & 26.420 \\
\hline 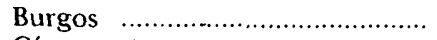 & 1.209 & 3.663 & 1.130 & 2.533 \\
\hline Cáceres & 5.54 & 3.222 & 418 & 2.804 \\
\hline 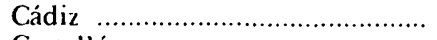 & 1.229 & 7.599 & 1.493 & 6.106 \\
\hline Castellón & 763 & 5.100 & 1.378 & 3.722 \\
\hline Ciudad Real & 328 & 2.840 & 483 & 2.357 \\
\hline 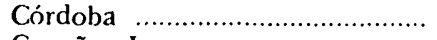 & 2.068 & 7.273 & 2.403 & 4.870 \\
\hline 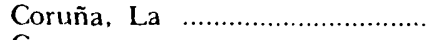 & 1.308 & 4.616 & 1.822 & 2.794 \\
\hline Cuenca & 378 & 1.939 & 377 & 1.562 \\
\hline Gerona & 454 & 2.613 & 1.012 & 1.601 \\
\hline 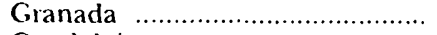 & 2.151 & 7.284 & 2.280 & 5.004 \\
\hline 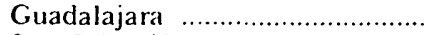 & 448 & 1.753 & 528 & 1.225 \\
\hline 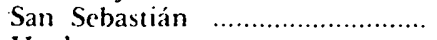 & 1.092 & 5.33 .3 & 1.925 & 3.408 \\
\hline Huelva & 818 & 5.762 & 1.212 & 4.550 \\
\hline 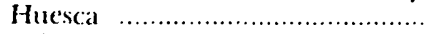 & 390 & 1.796 & 467 & 1.329 \\
\hline 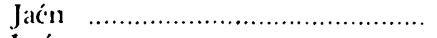 & 648 & 3.325 & 652 & 2.67 .3 \\
\hline 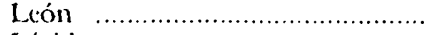 & 1.161 & 3.041 & 1.092 & 1.949 \\
\hline Lérida & 872 & 3.469 & 955 & 2.514 \\
\hline L.ogroño & 681 & 3.181 & 929 & 2.2 .52 \\
\hline 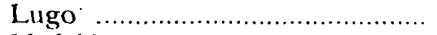 & 525 & 1.269 & 858 & 411 \\
\hline Madrid & 23.536 & 83.674 & 23.466 & 60.208 \\
\hline 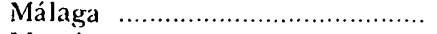 & 3.260 & 1.3 .050 & 4.274 & 8.776 \\
\hline Murcia & 2.819 & 7.501 & 2.814 & 4.687 \\
\hline 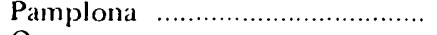 & 1.14 .3 & 6.236 & 1.935 & 4.301 \\
\hline Orense & .646 & 1.854 & 1.005 & 849 \\
\hline Oviedo & 1.265 & 4.393 & 2.695 & 1.698 \\
\hline 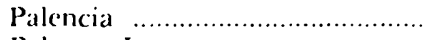 & 623 & 2.471 & 780 & 1.691 \\
\hline 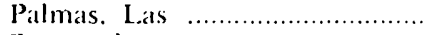 & 2.546 & 8.280 & 2.6 .58 & 5.622 \\
\hline 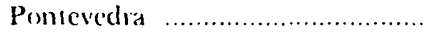 & 433 & 1.555 & 579 & 976 \\
\hline Salamanca …................................. & 1.6 .52 & 4.643 & 1.420 & 3.223 \\
\hline Santa Coruy de Tencrife ............. & 1.241 & 4.937 & 1.647 & 3.290 \\
\hline 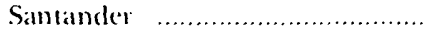 & 1.167 & 5.974 & 1.943 & 4.031 \\
\hline 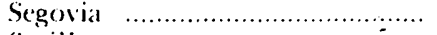 & 669 & 1.833 & 483 & 1.350 \\
\hline 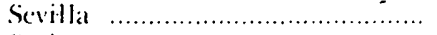 & 4.752 & 26.917 & 5.825 & 21.092 \\
\hline Sorial …n... & 343 & 1.031 & 268 & 763.3 \\
\hline Tartagona & 6.38 & 3.242 & 879 & 2.36 .3 \\
\hline 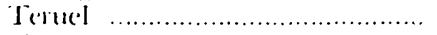 & 209 & 933 & 354 & 579 \\
\hline 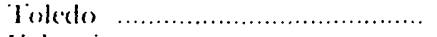 & 670 & 4.517 & 582 & 3,935 \\
\hline 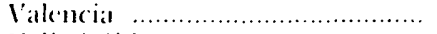 & 5.380 & 27.340 & 8.485 & 18.855 \\
\hline 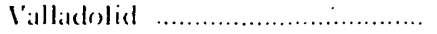 & 2.298 & 8.278 & 2.048 & 6.230 \\
\hline 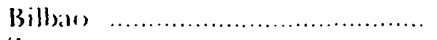 & 3.039 & $8.656 j$ & 3.118 & 5.538 \\
\hline 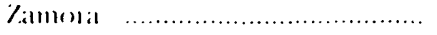 & 782 & 2.444 & 455 & 1.989 \\
\hline 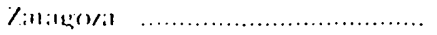 & 1.732 & 11.845 & 4.361 & 7.18 .1 \\
\hline
\end{tabular}


ESTADISTICA

3. MOVIMIENTO NATURAL DE LA POBLACION. 1978

\begin{tabular}{|c|c|c|c|c|}
\hline \multirow[b]{3}{*}{ En conjunto $\ldots \ldots \ldots \ldots \ldots \ldots \ldots$} & \multirow[t]{2}{*}{ Nuprialidad } & Natalidad & Mortalidad & $\begin{array}{c}\text { Crecimiento } \\
\text { vegetalivo }\end{array}$ \\
\hline & & \multicolumn{3}{|c|}{ Coefirientes por mil habitantes } \\
\hline & 6,94 & 17,06 & 7,82 & 9,24 \\
\hline 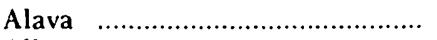 & 6,62 & 16,28 & 6,46 & 9,82 \\
\hline Albacete & 7,62 & 18,11 & 9,37 & 8,74 \\
\hline Alicante & 7,08 & 17,75 & 8,00 & 9,75 \\
\hline 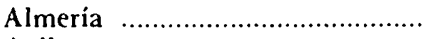 & 7,40 & 19,60 & $8,3 i$ & 11,23 \\
\hline 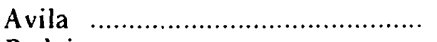 & 6,50 & 11,78 & 9,73 & 2,05 \\
\hline 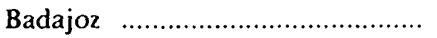 & 7,23 & 16,54 & 10,12 & 6,42 \\
\hline 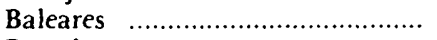 & 5,72 & 14,71 & 7,99 & 6,72 \\
\hline 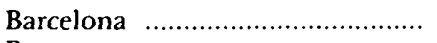 & 5,85 & 16,16 & 6,35 & 9,81 \\
\hline 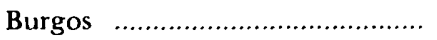 & 6,69 & 14,64 & 8,08 & 6,56 \\
\hline 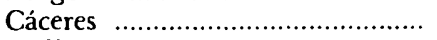 & 8,29 & 13,91 & 9,38 & 4,53 \\
\hline 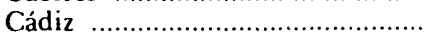 & 7,94 & 21,58 & 6,94 & 14,64 \\
\hline Castellón ................................... & 7,60 & 15,80 & 9,93 & 5,87 \\
\hline 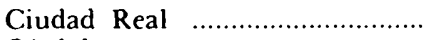 & 6,82 & 15,54 & 9,54 & 6,00 \\
\hline 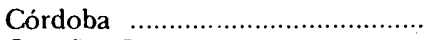 & 7,19 & 17,61 & 8,17 & 9,44 \\
\hline 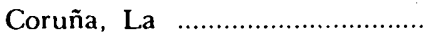 & 7,30 & 16,89 & 9,01 & 7,88 \\
\hline Cuenca & 6,93 & 12,00 & 10,46 & 1,54 \\
\hline 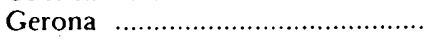 & 8,28 & 16,40 & 9,63 & 6,77 \\
\hline Granada & 7.47 & 18,61 & 8,05 & 10,56 \\
\hline 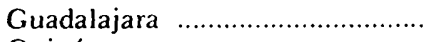 & 5,51 & 13,08 & 9,46 & 3,62 \\
\hline 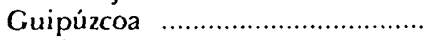 & 5,69 & 14,92 & 5,73 & 9,19 \\
\hline 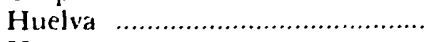 & 7,71 & 19,08 & 9,73 & 9,35 \\
\hline 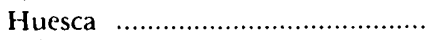 & 5,81 & 11,32 & 8,78 & 2,54 \\
\hline 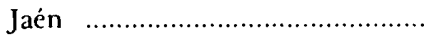 & 7,61 & 16,09 & 8,15 & 7,94 \\
\hline 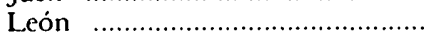 & 8,03 & 14,06 & 9,19 & 4,87 \\
\hline 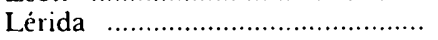 & 6,91 & 15,00 & 9,80 & 5,20 \\
\hline 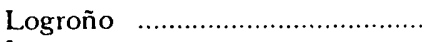 & 7,52 & 16,27 & 9,00 & 7,27 \\
\hline 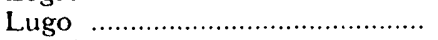 & 6,96 & 11,82 & 11,92 & $-0,10$ \\
\hline 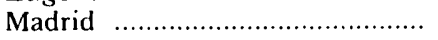 & 6,64 & 18,66 & 6,07 & 12,59 \\
\hline 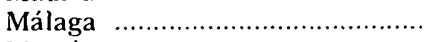 & 7,24 & 19,43 & 8,10 & 11,33 \\
\hline 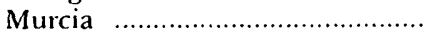 & 7,62 & 21,13 & 8,31 & 12,82 \\
\hline 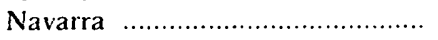 & 7.17 & 15,81 & 8,56 & 7,25 \\
\hline Orense & 6,32 & 11,44 & 10,50 & 0,94 \\
\hline Oviedo & 6,89 & 14,56 & 8,35 & 6,21 \\
\hline Palencia ..................................... & 7,95 & 14,03 & 10,10 & 3,93 \\
\hline 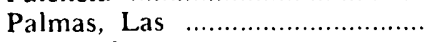 & 6,95 & 17,66 & 5,59 & 12,07 \\
\hline 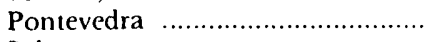 & 7,19 & 18,52 & 7,77 & 10,75 \\
\hline 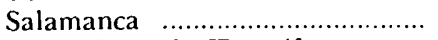 & 7,87 & 14,85 & 9.54 & 5,31 \\
\hline Santa Cruz de Tenerife ........... & 6,20 & 16,11 & 5,98 & 10,13 \\
\hline Santander & 7,73 & 17,37 & 7,76 & 9,61 \\
\hline 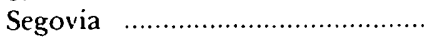 & 7,85 & 13,48 & 9,58 & 3,90 \\
\hline 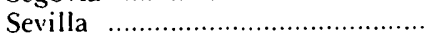 & 7,94 & 22,08 & 7,88 & 14,20 \\
\hline Soria & 6,21 & 10,80 & 10,14 & 0,66 \\
\hline 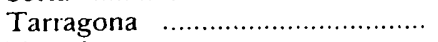 & 6,97 & 16,84 & 9,46 & 7,38 \\
\hline 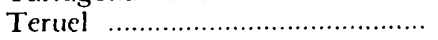 & 5,96 & 10,90 & 11,25 & $-0,35$ \\
\hline 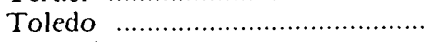 & 7,42 & 15,68 & 9,46 & 6,22 \\
\hline 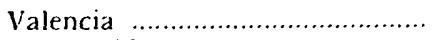 & 7,39 & 18,39 & 8,85 & 9,54 \\
\hline 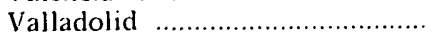 & 6,72 & 18,01 & 6,70 & 11,31 \\
\hline 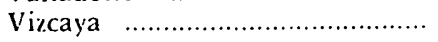 & 6,43 & 16,90 & 6,53 & 10,37 \\
\hline Zamora & 7,36 & 12,17 & 10,56 & 1,61 \\
\hline 7aragoza & 7,12 & 14.74 & 8,40 & 6,34 \\
\hline 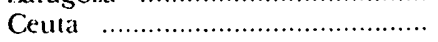 & 6,23 & 18,48 & 7,47 & 11.01 \\
\hline 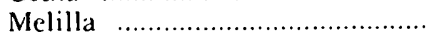 & 5.70 & 14,74 & 7.34 & 7,40 \\
\hline
\end{tabular}


REVL-1980, núm. 205. BALLESTER ROS, IGNACIO. EL MOVIMIENTO NATURAL DE LA POBLACI...

4. MOVIMIENTO NATURAL DE LA POBLACION. 1978

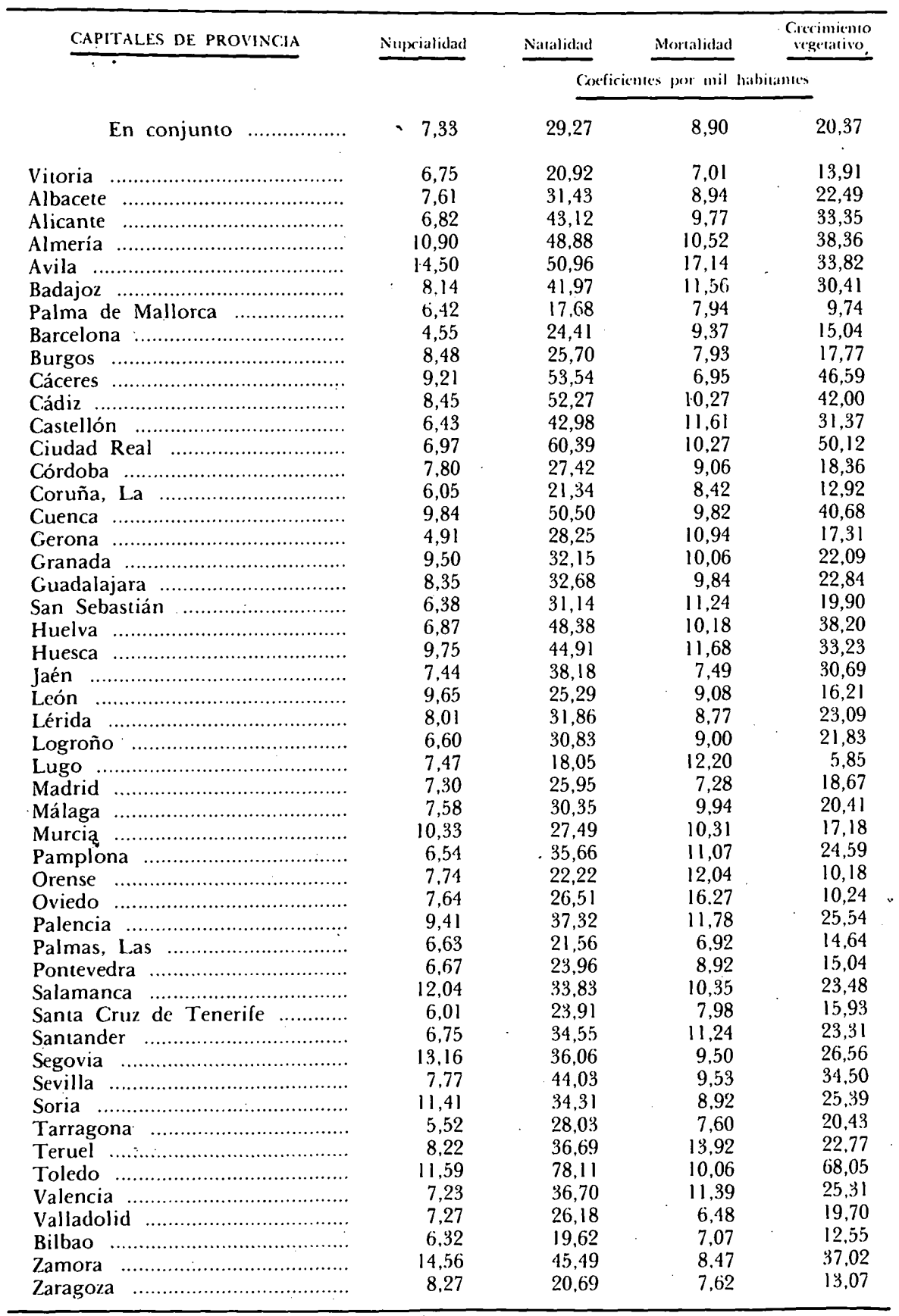


REVL-1980, núm. 205. BALLESTER ROS, IGNACIO. EL MOVIMIENTO NATURAL DE LA POBLACI...

REVISTA

$\mathrm{DE}$

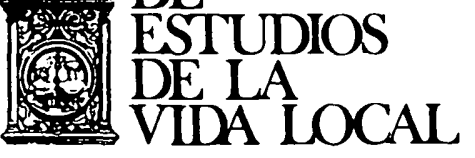

IV. JURISPRUDENCIA

REVL-1980, núm. 205. BALLESTER ROS, IGNACIO. EL MOVIMIENTO NATURAL DE LA POBLACI... 
REVL-1980, núm. 205. BALLESTER ROS, IGNACIO. EL MOVIMIENTO NATURAL DE LA POBLACI...

REVL-1980, núm. 205. BALLESTER ROS, IGNACIO. EL MOVIMIENTO NATURAL DE LA POBLACI... 